\title{
Josephson current in strongly correlated double quantum dots
}

\author{
Rok Žitko, ${ }^{1}$ Minchul Lee, ${ }^{2}$ Rosa López, ${ }^{3}$ Ramón Aguado, ${ }^{4}$ and Mahn-Soo Choi ${ }^{5}$ \\ ${ }^{1}$ J. Stefan Institute, Jamova 39, SI-1000 Ljubljana, Slovenia \\ ${ }^{2}$ Department of Physics, Kyung Hee University, Yongin 446-701, Korea \\ ${ }^{3}$ Departament de Física, Universitat de les Illes Balears, E-07122 Palma de Mallorca, Spain \\ ${ }^{4}$ Teoría de la Materia Condensada, Instituto de Ciencia de Materiales de Madrid (CSIC) Cantoblanco,28049 Madrid, Spain \\ ${ }^{5}$ Department of Physics, Korea University, Seoul 136-701, Korea
}

(Dated: November 1, 2018)

\begin{abstract}
We study the transport properties of a serial double quantum dot (DQD) coupled to two superconducting leads, focusing on the Josephson current through the DQD and the associated 0- $\pi$ transitions which result from the subtle interplay between the superconductivity, the Kondo physics, and the inter-dot superexchange interaction. We examine the competition between the superconductivity and the Kondo physics by tuning the relative strength $\Delta / T_{K}$ of the superconducting gap $\Delta$ and the Kondo temperature $T_{K}$, for different strengths of the superexchange coupling determined by the interdot tunneling $t$ relative to the dot level broadening $\Gamma$. We find strong renormalization of $t$, a significant role of the superexchange coupling $J$, and a rich phase diagram of the 0 and $\pi$-junction regimes. In particular, when both the superconductivity and the exchange interaction are in close competion with the Kondo physics $\left(\Delta \sim J \sim T_{K}\right.$ ), there appears an island of $\pi^{\prime}$-phase at large values of the superconducting phase difference.
\end{abstract}

PACS numbers: 73.23.-b,72.15.Qm,74.45.+c

Introduction.- In a metal containing a dilute concentration of magnetic impurities the competititon between Kondo physics, which favours screening of the localized spins by the itinerant conduction-band electrons [1], and antiferromagnetic (AF) exchange interactions between impurities leads to a quantum phase transition [2]. Even more interesting properties emerge when the metal turns superconducting below the critical temperature. For s-wave superconductors, Cooper pairs formed by itinerant electrons [3] are yet another possible singlet state which competes with the above. The intriguing interplay of these phenomena, which might actually coexist in complex materials such as heavy-fermion superconductors, governs the low temperature physics of these systems. Nanoscale systems allow to tune the ratio between the relevant parameters (the Kondo temperature $T_{K}$, the AF exchange interaction $J$, and the superconducting gap $\Delta$, respectively) and, therefore, enable thorough investigations of such competition in a controlled setting. In the simplest case of single quantum dots attached to superconducting reservoirs, where only Kondo physics and superconductivity are relevant, a sign change of the Josephson current, from positive 0 -junction to negative $\pi$-junction behavior, signals a quantum phase transition between a singlet and a doublet ground state as $T_{K} / \Delta$ decreases [4-6]. This 0 to $\pi$-junction transition has been experimentally realized, confirming some of these physical aspects [7]. A double quantum dot (DQD) coupled to normal metals constitutes a physical realization of the twoimpurity Kondo model [8-[10], as demonstrated experimentally [11, 12]. When the reservoirs become superconducting, this system is a minimal artificial realization of the described competition among three different spin-singlet ground states. In this Letter we focus on a detailed analysis of the Josephson current which, as a ground state property, shows signatures of this subtle competition. Our results, obtained by a highly efficient numerical renormalization group (NRG) scheme, able to deal with superconducting correlations, are summarized in Figs. 2 and 3 The interplay between superconductivity and the Kondo physics is studied by tuning the relative strength $\Delta / T_{K}$. The role of the superexchange coupling is tuned by the interdot tunneling $t$ relative to the dot level broadening $\Gamma$. Importantly, we find strong renormalizations of $t$ and a significant role of the superexchange coupling $J$ compared with the previous works[8, 9, 13] based on the slave-boson mean-field theory (SBMFT). Moreover, we find a rich phase diagram of the $0-\pi$ transition. In particular, when both the superconductivity and the superexchange are in close competion with the Kondo physics ( $\Delta \sim J \sim T_{K}$ ), there appears an unexpected island of $\pi^{\prime}$-phase at large values of the superconducting phase difference $\phi$. We provide clear interpretations by examining the spin-state-resolved Andreev bound states inside the superconducting gap.

Model._ The system that we consider is a DQD modelled as a two-impurity Anderson model connected to two superconducting leads (Fig. 1) described by standard BCS Hamil-

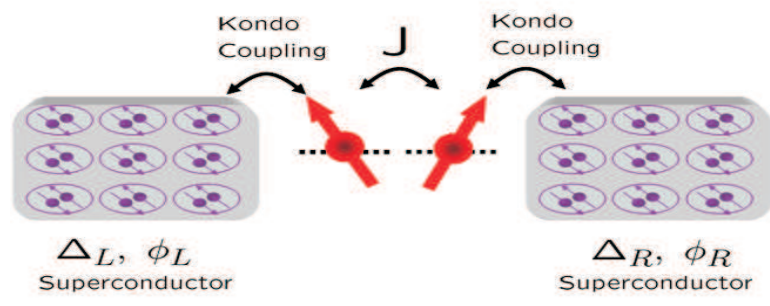

FIG. 1: (color online) Schematics of the DQD system coupled to superconducting leads. In the deep Kondo limit, this system is an artificial realization of the two-impurity Kondo problem in the presence of superconducting correlations. 
tonians [3]: $\mathcal{H}=\mathcal{H}_{\mathrm{D}}+\mathcal{H}_{\mathrm{L}}+\mathcal{H}_{\mathrm{T}}$, where

$$
\begin{aligned}
& \mathcal{H}_{\mathrm{D}}=\sum_{i}\left(\epsilon n_{i}+U n_{i \uparrow} n_{i \downarrow}\right)-t \sum_{\mu}\left(d_{1 \mu}^{\dagger} d_{2 \mu}+(h . c .)\right) \\
& \mathcal{H}_{\mathrm{L}}=\sum_{\ell \mathbf{k}}\left[\epsilon_{\mathbf{k}} n_{\ell \mathbf{k}}-\left(\Delta_{\ell} e^{i \phi_{\ell}} c_{\ell \mathbf{k} \uparrow}^{\dagger} c_{\ell-\mathbf{k} \downarrow}^{\dagger}+(\text { h.c. })\right)\right] \\
& \mathcal{H}_{\mathrm{T}}=V \sum_{\ell \mathbf{k} \mu}\left[c_{\ell \mathbf{k} \mu}^{\dagger} d_{\ell \mu}+(\text { h.c. })\right] .
\end{aligned}
$$

Here $c_{\ell \mathbf{k} \mu}$ describes an electron with energy $\epsilon_{\mathbf{k}}$, momentum $\mathbf{k}$, and spin $\mu$ on the lead $\ell=1,2$, and $d_{i \mu}$ an electron in the dot $i=1,2 ; n_{\ell \mathbf{k}} \equiv \sum_{\mu} c_{\ell \mathbf{k} \mu}^{\dagger} c_{\ell \mathbf{k} \mu}$ and $n_{i} \equiv \sum_{\mu} d_{i \mu}^{\dagger} d_{i \mu}$. $\epsilon$ is the single-particle energy on each dot that is tuned by gate voltages and $U$ is the on-site Coulomb interaction. The electrons can tunnel between the two dots with the tunneling amplitude $t . \Delta_{\ell}$ is the superconducting gap, and $\phi_{\ell}$ the phase of the superconducting order parameter.

The two leads are assumed to be identical except for the superconducting phases. Assuming that the leads have a flat band with the density of states $\rho=1 / 2 D$, where $2 D$ is the bandwidth, the hybridization between the dots and the leads is well characterized by a single parameter $\Gamma=\pi \rho V^{2}$. Since we are interested in the Kondo correlations, we concentrate on the Kondo regime with localized level $-\epsilon \gg \Gamma$ and large charging energy $U \geq 2|\epsilon|$. For the representative results shown below, we choose $\Gamma=0.014 D$ or $\Gamma=0.02 D$, fix $\epsilon=-0.2 D$, and take the large $U=\infty$ limit. The parameter space is nevertheless still large. We examine the results by varying $\Delta / T_{K}$, $t / \Gamma$, and $\phi \equiv \phi_{L}-\phi_{R}$.

We solve the Hamiltonian using the numerical renormalization group (NRG) method [14]. Due to the relatively low symmetry of the nrohlem (in narticular due to the lack of charoe

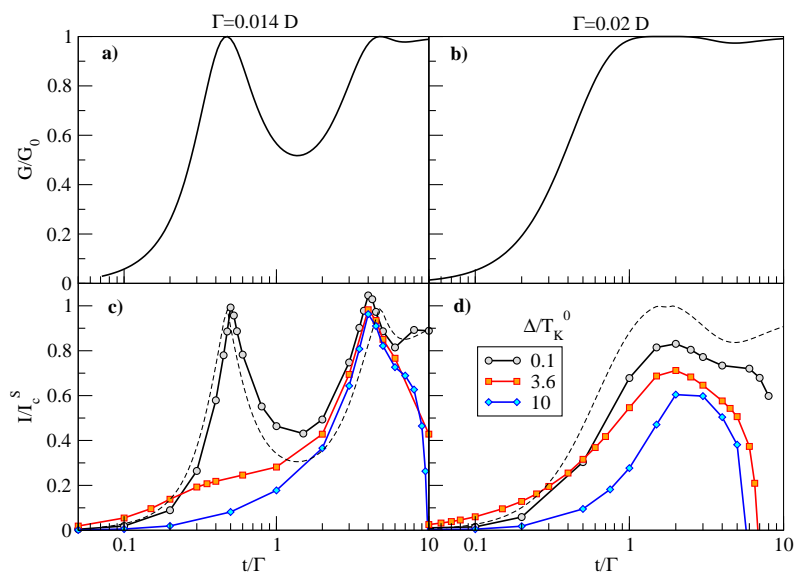

FIG. 2: (color online) Normal state conductance $(a, b)$ and the critical Josephson current in the superconducting state $(\mathrm{c}, \mathrm{d})$ as a function of $t / \Gamma$ for $\Gamma=0.014 D(\mathrm{a}, \mathrm{c})$ and $\Gamma=0.02 D(\mathrm{~b}, \mathrm{~d})$. The results are expressed in units of the conductance quantum $G_{0}=2 e^{2} / h$ and the supercurrent quantum $I_{c}^{s}=e \Delta / \hbar$. In the superconducting case (c,d), the different curves are for $\Delta / T_{K}=0.1$ (black circle), 3.6 (red square), and 10 (blue diamond). The dashed line is from an effective non-interacting theory, Eq. (4). conservation), the NRG iteration is numerically very demanding and special attention is necessary to obtain reliable results. Using a new discretization scheme the numerical artifacts due to a large discretization parameter $\Lambda$ are almost completely canceled out; the technical details are given elsewhere.

We first review two crucial effects from a previous work on DQD with normal leads [10] which keep playing significant roles in the present superconducting case. Firstly, the interdot tunneling $t$ is significantly renormalized compared with the predictions based on the SBMFT [8, 9, 13]. It is important to treat this effect properly to fully account for the transport. Secondly, the inter-dot antiferromagnetic superexchange $J$ is finite even for $U=\infty$.[10] This interaction is mediated by the virtual tunneling of electrons to the conduction leads. It is thus important to take into account the interplay of the superexchange coupling with the superconductivity and the Kondo effect.

Strong coupling limit $\left(T_{K} \gg \Delta\right)$. - Figure 2 shows normal state conductance (a,b) and the critical Josephson current (c,d). Here we focus on the strong coupling limit, $\Delta / T_{K}=$ 0.1 (black circles). In this limit, the critical Josephson current $I$ shows similar features as the normal-state conductance. It peaks at equal values of $t / \Gamma$ and, remarkably, when the conductance in the normal state is unitary, the Josephson current reaches the quantum limit $I_{c}^{s}=e \Delta / \hbar$. This is expected since the Kondo effect dominates over the superconductivity, therefore the transport is determined by the competition between the Kondo physics and the interdot superexchange (for $t / \Gamma<5)$ or interdot molecular orbitals $(t / \Gamma>5)$. As we analyzed in detail in the previous work for the normal lead case, the peaks in $G$ and $I_{c}$ at $t / \Gamma \approx 0.4$ (for $\Gamma=0.014 D$ ) result from the crossover from the "Kondo singlet" to the "superexchange singlet": For $t / \Gamma<0.4, J<T_{K}$, wheares for $0.4<t / \Gamma<5, J>T_{K}$. We stress that the crossover is shifted significantly to smaller $t / \Gamma=0.4$ compared with the estimation from SBMFT, [8, 9, 13] due to the previously mentioned strong renormalization of $t$. As $t / \Gamma$ increases beyond 5 , the DQD starts to form molecular orbitals and effectively behaves as a single QD. In this regime, the Josephson current in single dots has $\pi$ shift in the Coulomb blockade regime [46], which is directly related to the $\pi$-phase in Fig. 3 $(a, b)$ for large $t / \Gamma$.

For comparison, we have also calculated the critical Josephson current as $I=\max _{\phi} I(\phi)$, where [15]

$$
\frac{I(\phi)}{I_{c}^{s}}=\frac{g}{2} \sin \phi \sqrt{1-g \sin ^{2}(\phi / 2)},
$$

where $I_{c}^{s}=e \Delta / \hbar$ is the critical current of a transparent single-mode junction, and $g=G / G_{0}$ is the (dimensionless) normal-state conductance obtained from a NRG calculation. These relations are applicable only under the assumption that the QD state is weakly affected by the superconductivity. As can be seen in Fig. 2 for $t / \Gamma \lesssim 5$, the result from the effective theory (dashed lines) and the full NRG calculation show a qualitative agreement. For larger $t(t / \Gamma>5)$, we enter the 


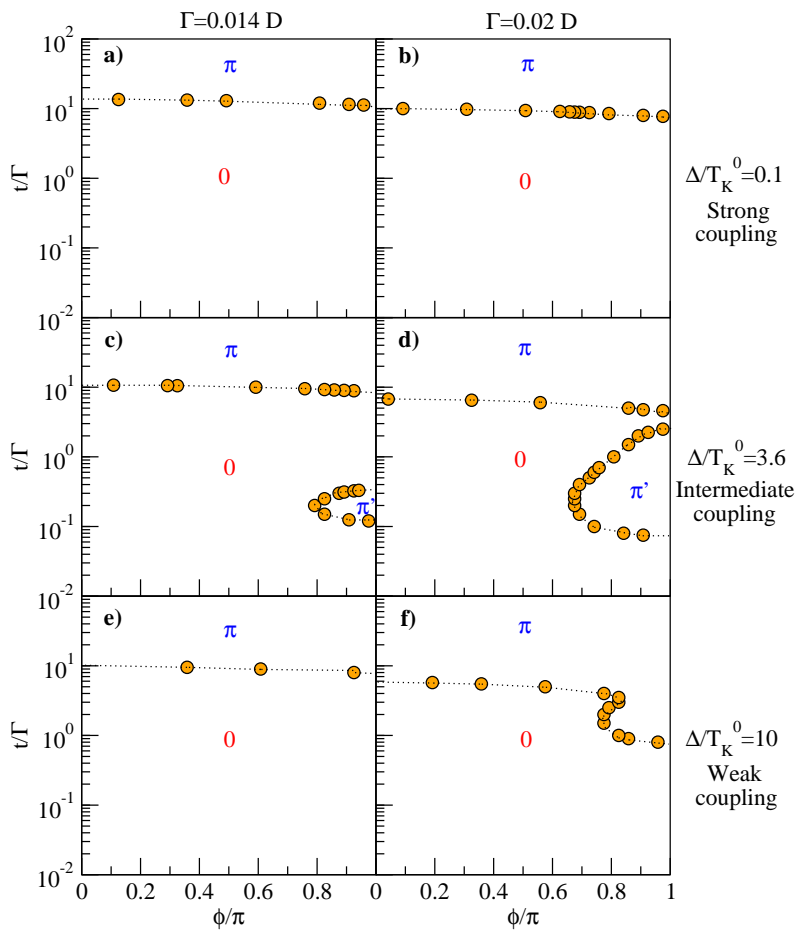

FIG. 3: Phase boundaries between the 0 - and $\pi$-states of the Josephson current for $\Gamma=0.014 D$ (left panels) and $\Gamma=0.02 D$ (right panels). When both the inter-dot superexchange coupling and the superconductivity are in close competition with the Kondo effect between the superconducting leads and adjacent dots $\left(t / \Gamma \sim 0.2, \Delta \sim T_{K}\right)$, there appears an island of $\pi^{\prime}$-phase at larger phase difference.

single-dot regime and the Kondo effect is suppressed. This leads to the deviation of the two results.

Weak coupling limit $\left(T_{K} \ll \Delta\right)$. - The results, shown in Figs. 2 and 3 for $\Delta / T_{K}=10$ (gray diamonds, blue online), indicate that the superconducting correlations in the leads suppress the Kondo effect and the Josephson current remains small until the system enters the single-dot regime. It is remarkable that the S-DQD-S system behaves as a 0 -junction in the weak coupling limit in contrast to the S-QD-S case where the $\pi$-junction appears in the same regime. In single quantum dots, the appearance of $\pi$-junction is due to the reversal of the order of the electrons forming Cooper pairs after tunneling. [4] However, in series coupled DQD, the order is preserved so that no additional phase factor arises from the tunneling and thus 0-junction is formed even in the Coulomb blockade regime. Specifically, the perturbation theory applied in the weak coupling limit for $U \rightarrow \infty$ gives

$$
\begin{array}{r}
\frac{I}{I_{c}^{s}}=\sin \phi \sum_{\mathbf{k q}} \frac{2 \Delta t^{2} t_{\mathbf{k}}^{2} t_{\mathbf{q}}^{2}}{E_{\mathbf{k}} E_{\mathbf{q}}\left[\left(\epsilon-E_{\mathbf{q}}\right)^{2}-t^{2}\right]\left[\left(\epsilon-E_{\mathbf{k}}\right)^{2}-t^{2}\right]} \\
\times\left(\frac{1}{E_{\mathbf{q}}+E_{\mathbf{k}}}+\frac{1}{2|\epsilon|}\right)
\end{array}
$$

with $E_{\mathbf{k}} \equiv \sqrt{\epsilon_{\mathrm{k}}^{2}+\Delta^{2}}$. Our NRG calculations confirm that the ground state is a spin singlet as long as the 0 -junction is

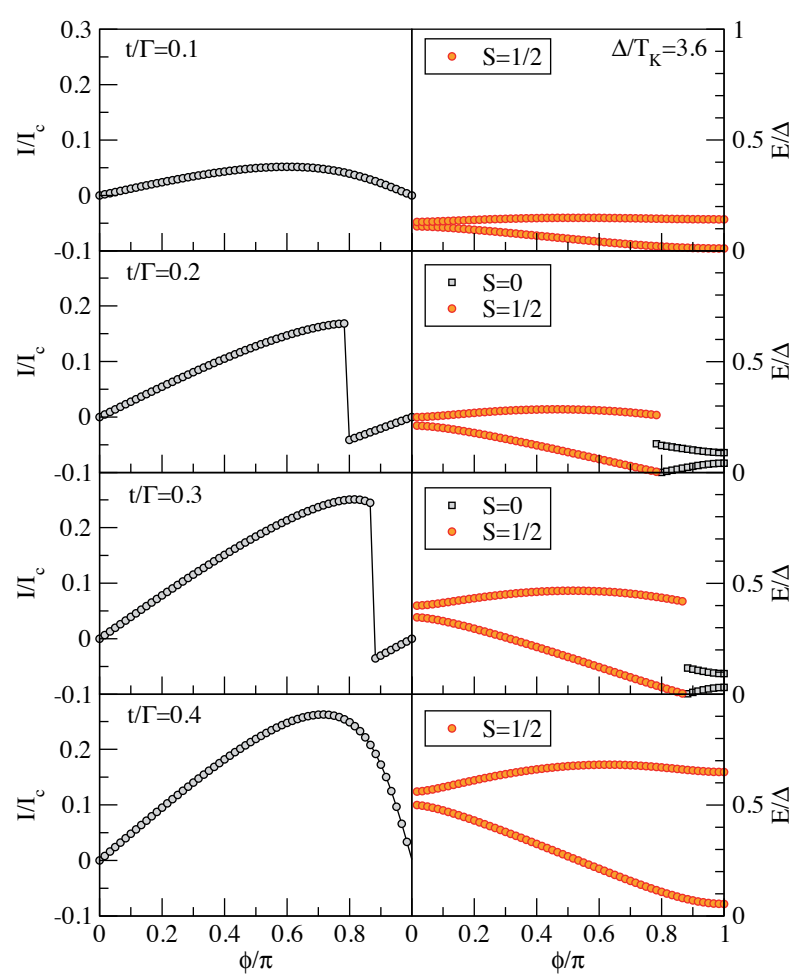

FIG. 4: (color on-line) Left panels: Josephson current vs phase difference near the $\pi^{\prime}$-phase $\left(\Delta / T_{K}=3.6\right.$ and $t / \Gamma=$ $0.1,0.2,0.3,0.4$, from top to bottom). Right panels: Corresponding spin-state-resolved Andreev bound states inside the superconducting gap. Spin singlet states are depicted by (red) circles and doublets by (black) squares. The changes in the spin states are closely related to the island of $\pi^{\prime}$ phase in Fig. 3 (c) [similar spin-state-resolved Andreev states for corresponding parameters will also explain the island in Fig. 3(d)].

formed. Hence, in contrast to the single quantum dot system, in a large part of the parameter space there exists no phase transition as we move from the weak to the strong coupling limits by varying $\Delta / T_{K}$ : we always have a spin singlet state with 0 -junction behavior.

Subsequent transition into $\pi$-junction for very large $t / \Gamma$ in Fig. 3 (e,f) is ascribed to the competition between effective spin-1/2 Kondo correlation and superconductivity as in the strong coupling limit. Since the superconducting gap $\left(\Delta / T_{K}^{0} \gg 1\right)$ is larger than in the strong coupling limit $\left(\Delta / T_{K}^{0} \ll 1\right)$, however, the transition can take place at smaller values of $t / \Gamma$ for which the effective Kondo temperature $T_{K}$ is higher.

Moreover, the critical current is relatively large even though the system is in the weak coupling limit, while in the single quantum dot the critical current in this limit is very small $\left(I / I_{c}^{s}<0.1\right)[5]$. A very likely explanation is that the oneelectron spin- $1 / 2$ Kondo state is formed at smaller values of $t / \Gamma$ and that strong superconductivity is responsible for it. The (one-electron) Kondo assisted tunneling then makes the junction more transparent and enhances the critical current. Hence, the physical origin of the peak in the critical current is 
different in the weak and strong coupling limits.

Intermediate coupling $\left(T_{K} \sim \Delta\right)$. - A highly non-trivial behavior occurs for $T_{K} \sim \Delta$. In this regime, the superconductivity, the superexchange, and the Kondo physics can all be in close competion. This subtle interplay keeps the Josephson critical current finite, somewhere between the weak coupling and strong coupling limit, Fig. 2 (c and d), except for very large $t / \Gamma$, where single-dot physics again governs the transport.

More interestingly, the phase diagrams in Fig. 3 (c,d) reveal the reentrance behavior from the 0 -junction, to $\pi^{\prime}$-junction, and back to 0 -junction, and eventually to the $\pi$-junction for larger superconducting phase difference $\phi$. In order to understand this behavior, we closely examine the subgap Andreev bound states, which are Bogoliubov quasi-particle excitations from the ground state[3] and whose derivatives with respect to $\phi$ give the Josephson current [16]. In Fig. 4 we plot the energy levels of the Andreev states as a function of $\phi$, in the parameter regime corresponding to the island in Fig. 3 (c). Let us focus on, say, $\phi=0.9 \pi$. For $t / \Gamma<0.1$, the singlet Kondo clouds are formed between the superconductors and the adjacent dots, thus the ground state is likewise a spin singlet, while the excitations correspond to doublet states, see Fig. 4 (b). As the Josephson current is given approximately by the phasedifference-derivative of the Andreev levels, this corresponds to the 0 -junction behavior; Fig. 4 (a). For $0.4<t / \Gamma<5$, the local inter-dot singlet state is induced on the DQD due to the antiferomagnetic superexchange interaction, thus the ground state is again a spin singlet; Fig. 4(h). As before, this results in the 0 -junction behavior; Fig. 4 (g).

In the above two cases, both Kondo effect and superexchange barely win over the superconductivity, for all values of $\phi$. However, when $0.1<t / \Gamma<0.4$, the Kondo effect is suppressed by the large phase difference. [5] This is indicated in the fact that the ground state is now a doublet, while the excited state is a singlet, as shown in Fig. 4 (d,f). Accordingly, the transport properties are different and, in particular, the $\pi$ junction behavior is observed; see Fig. 4 (c,e). This regime is denoted as $\pi^{\prime}$ in the phase diagram in Fig. 3. While the $\pi$ phase for large $t$ corresponds to the single occupancy of the dots, the $\pi^{\prime}$ phase occurs for the double occupancy. For large values of $\Gamma(\Gamma=0.02 D)$, the $\pi^{\prime}$ island becomes bigger and it can merge with the $\pi$ regime.

Conclusion.- We have studied the Josephson current through a serial double quantum dot coupled to two superconducting leads. We have observed a strong renormalizations of $t$, the significant role of the superexchange coupling $J$, and a rich phase diagram featuring different $0-\pi$ transitions. In particular, when both the superconductivity and the superexchange were in close competion with the Kondo physics ( $\Delta \sim J \sim T_{K}$ ), there appeared an island of $\pi^{\prime}$-phase at larger values of the superconducting phase difference. This finding motivates further studies of this regime, which may shed new light on the physics of heavy-fermion superconductors.
M.-S.C. was supported by the NRF grant (2009-0080453), the BK21 program, and the APCTP. R. A. was supported by MEC-Spain (Grant No. MAT2006-03741, FIS2009-08744), CSIC and CAM (Grant No. CCG08- CSIC/MAT- 3775) M.S.C. and R.A. acknowledge useful discussions with A. Levy Yeyati. R. Z. was supported by ARRS grant no. Z1-2058.

[1] A.C. Hewson, The Kondo Problem to Heavy Fermions (Cambridge University Press, Cambridge, UK, 1993)

[2] B. A. Jones, C. M. Varma, and J. W. Wilkins, Phys. Rev. Lett. 61, 125 (1988).

[3] M. Tinkham, Introduction to Superconductivity (McGraw-Hill, New York 2nd ed. 1996).

[4] I.O-. Kulik, Zh. ksp. Teor. Fiz. 49, 585 (2000).[Sov. phys. JETP 22, 841 (1966)]. H. Shiba and T. Soda, Prog. Teor. Phys. 41, 25 (1969). L.I. Glazman and K.A. Matveev, Pis'ma Zh. ksp. Teor. Fiz. [JETP Lett. 49, 659 (1989)]. B.I. Spivak and S.A. Kilvelson, Phys. Rev. B 43, R3740 (1991).

[5] M.S. Choi, M. Lee, K. Kang, and W. Belzig, Phys. Rev. B 70, 020502(R) (2004); ibib Phys. Rev. Lett 94, 229701 (2005). F. Siano and R. Egger, Phys. Rev. Lett. 93, 047002 (2004); Phys. Rev. Lett 94, 229702 (2005). E. Vecino, A. MartinRodero, and A.L. Yeyati, Phys. Rev. B 68, 035105 (2003).

[6] A. Zazunov, A. Schulz, and R. Egger, Phys. Rev. Lett. 102, 047002 (2009). T. Meng, P. Simon, and S. Florens Phys. Rev. B 79134513 (2009). C. Karrasch, A. Oguri, and V. Meden, Phys. Rev. B 77024517 (2008). G. Sellier et al., Phys. Rev. B 72174502 (2005). A.L. Yeyati, A. Martin-Rodero, and E. Vecino, Phys. Rev. Lett. 91, 266802 (2003). Y. Avishai, A. Golub, and A.D. Zaikin, Phys. Rev. B 67, 041301(R) (2003). A.V. Rozhkov and D.P. Arovas, Phys. Rev. B 62, 6687 (2000). A.V. Rozhkov, D.P. Arovas, F. Guinea, Phys. Rev. B 64, 233301 (2001). A. A. Clerk and V. Ambegaokar, and S. Hershfield, Phys. Rev. B 61, 3555 (2000).

[7] J.-P. Cleuziou et al., Nature Nanotechnology 1, 53 (2006); A. Eichler et al., Phys. Rev. B 79, 161407R (2009).

[8] A. Georges and Y. Meir, Phys. Rev. Lett. 82, 3508 (1999).

[9] R. Aguado and D.C. Langreth, Phys. Rev. Lett. 85, 1946 (2000), ibib Phys. Rev. B 67, 245307 (2003). T. Aono and M. Eto, Phys. Rev. B 63, 125327 (2001). R. Lopez, R. Aguado, and G. Platero, Phys. Rev. Lett. 89, 136802 (2002). P. Simon, R. Lopez, and Y. Oreg et al., 94, 086602 (2005).

[10] M. Lee, M.-S. Choi, R. López, R. Aguado, J. Martinek, \& R. Žitko, arXiv:0911.0959

[11] N.J. Craig et al., Science 304565 (2004).

[12] H. Jeong et al., Science 293, 2221 (2001). J.C. Chen, A.M. Chang, M.R. Melloch, Phys. Rev. Lett. 92, 176801 (2004).

[13] Bergeret, F. S., A. Levy Yeyati, \& A. Martin-Rodero, Phys. Rev. B 74 (13), 132505 (2006).

[14] K. G. Wilson, Rev. Mod. Phys. 47, 773 (1975); W. Hofstetter and G. Zarand, Phys. Rev. B 69, 235301 (2004). Rok Zitko and Thomas Pruschke, Phys. Rev. B 79, 085106 (2009).

[15] C. W. J. Beenakker and H. Van Houten. Single-electron tunneling and Mesoscopic devices, edited by $\mathrm{H}$. Koch, and $\mathrm{H}$. Lbbig (Springer Berlin 1992).

[16] In this qualitative discussion we ignore the small contribution from the continuum of states above the gap. 